\title{
Prevention of Oculocardiac Reflex by Premedication with Low Dose I/V Ketamine during Strabismus Surgery
}

\author{
MEHREEN AKRAM ${ }^{1}$, IQRA MUSHTAQ ${ }^{2}$, RAFIA KOUSAR ${ }^{3}$ \\ ${ }^{1}$ Consultant Anaesthesia, Hameed Latif Hospital, Lahore \\ ${ }^{2}$ Senior Registrar Anaesthesia, Mayo Hospital, Lahore \\ ${ }^{3}$ Senior Registrar Anaesthesia, Sahara Medical College, Narowal \\ Correspondence to Dr. Mehreen Akram, Consultant Anaesthesia, Email: drmehreenakram@gmail.com, Cell: 0321-7791894
}

\begin{abstract}
Background: OCR commonly occurs during strabismus surgery, producing bradycardia, arrhythmias and even cardiac arrest after manipulation of orbital structures. Ketamine is NMDA receptor antagonist and acts as an analgesic.

Aim: To determine the effect of ketamine premedication on prevention of OCR during strabismus surgery.

Study design: Randomized control trial

Methodology: 60 patients were randomly divided into two groups i.e., Ketamine 0.75mg/kg (Group K) and control (Group C). Group K patients were premedicated with $0.75 \mathrm{mg} / \mathrm{kg}$ ketamine while Group C patients did not receive any premedication. Heart rate and ECG were observed $30 \mathrm{sec}$ before and continuously after traction on extraocular muscles was applied upto end of surgery for bradycardia and arrhythmias. Percentage change in HR and presence of arrhythmias was documented. All the data was collected using case report form and analyzed using SPSS version 15.

Results: In Group C, 15(50\%) cases had arrhythmias while in Group K, only 4(13.33\%) cases had arrhythmias. Mean HR in Group C was $118.77 \pm 6.92 / \mathrm{min}$ and in Group K was $101.57 \pm 15.65 / \mathrm{min}$. In Group C, oculocardiac reflex was present in 23(76.7\%) cases and in Group K, OCR was present in $6(20 \%)$ cases and prevented in $24(80 \%)$ cases.

Conclusion: Premedication with $0.75 \mathrm{mg} / \mathrm{kg}$ IV ketamine significantly reduces the occurrence of oculocardiac reflex during strabismus surgery.

Keywords: Ketamine, Oculocardiac Reflex, Premedication, Prevention, Strabismus
\end{abstract}

\section{INTRODUCTION}

Strabismus is misalignment of optical axis. It is a frequently occurring disorder, corrected by surgery in which one or more extra-ocular muscles are shortened or lengthened. During strabismus corrective surgery, major anesthetic concern is oculocardiac reflex (OCR) especially in pediatric population ${ }^{1,2}$. A decrease in heart rate of more than $20 \%$ from baseline or development of any type of dysrhythmia after manipulation of orbital structures or pressure on the globe is known as oculocardiac reflex $(\mathrm{OCR})^{3-7}$. The incidence of OCR is $32-90 \%{ }^{1}$. It was initially described by Aschner and Dagnini in $1908^{2,4,8}$.

OCR occurs during traction on extra-ocular muscles, mainly medial rectus ${ }^{9,10}$, or pressure on the eyeball or its contents including periosteum ${ }^{1}, 2,11$. This manipulation produces cardiac dysrhythmias ranging from bradycardia and ventricular ectopy to ventricular fibrillation, AV block and sinus arrest ${ }^{1,4,11}$. In conscious patients, it is accompanied by nausea ${ }^{4}$. OCR is mediated by trigeminovagal reflex arc. The afferent limb comprises of ciliary nerve to the ciliary ganglion to ophthalmic division of trigeminal nerve and then to trigeminal sensory nucleus. The efferent limb consists of vagus nerve to heart ${ }^{8,12}$.

OCR is managed by asking the surgeon to stop stimulation, maintaining adequate anesthetic depth or pre-medication with anticholinergic agent ${ }^{2}$. Atropine is the most commonly used anticholinergic drug in clinical practice. Atropine blocks muscarinic acetylcholine receptors including those in SA node and AV node, resulting in increased firing from SA node and rapid conduction through AV node, ultimately producing tachycardia ${ }^{1,4}$. However, use of atropine is also controversial ${ }^{8}$. Atropine doesn't completely prevent OCR and can also convert bradycardia into PVCs, bigemini and ectopic beats and such arrhythmias are more persistant ${ }^{1}$. Furthermore, it can reduce cardiac ouput especially in infants and can also lead to central anticholinergic symptoms if it crosses the blood-brain barrier ${ }^{8}$.

Many anesthesiologists have reported ketamine to be better than other drugs for prevention of $\mathrm{OCR}^{1,2,11}$. Ketamine is NMDA receptor antagonist and acts as analgesic. It causes central stimulation of sympathetic system and inhibition of reuptake of

Received on 22-05-2021

Accepted on 13-09-2021 norepinephrine from nerve terminals ${ }^{1}$ and enhanced norepinephrine release ${ }^{14}$. Hence, it has cardiostimulatory properties $^{8,11}$ leading to positive chronotropic effect ${ }^{1,8}$.

Ketamine is associated with emergence reaction including vivid dreams, floating sensation, dizziness and blurred vision ${ }^{14}$. This reaction was exaggereated by using substantial doses of ketamine (higher than $2 \mathrm{mg} / \mathrm{kg}$ ) ${ }^{15}$. Therefore, in our study we used low dose ketamine to minimize its side effects as opposed to previous studies in which larger doses were utilized.

The purpose of our study is to measure the frequency of prevention of OCR after premedication with versus without low dose IV ketamine during strabismus surgery.

\section{MATERIALS AND METHODS}

On the basis of previous related study, 30 patients were allocated to each group (Group C and Group K). The sample size was calculated by taking expected frequency of OCR in control group (Group C) to be $63 \%$ and in ketamine group (Group K) to be $20 \%$ with $5 \%$ level of significance and $80 \%$ power of test. This randomized control study was done in Department of Anaesthesia, King Edward Medical University/Mayo Hospital, Lahore. Patients, aged 10-24 years of both sex undergoing strabismus surgery corresponding to American Society Of Anaesthesiologists Class I or II and those who gave consent to participate in the study, were included. Following patients were excluded from the study as per exclusion criteria:

- $\quad$ Patients having hypertension $(B P>140 / 90)$ or IHD

- Patients with history of conduction abnormalities on clinical examination

- Patients having known allergies to any of the anesthetic drugs being used

- $\quad$ Patients with mental illness (history/medical record)

- Patients with communication difficulties

By using random computer number, 60 patients were allocated into two equal groups (Group $\mathrm{K}$ and Group $\mathrm{C}$ ). Preoperative assessment was done a day before surgery. Study plan was described to the patient and his/her parents and informed consent taken. Anonymity and confidentiality was ensured. On the day of surgery, patients after NPO of 6 hours, were allocated to one of the groups by random table number. With non-invasive monitoring (heart rate, ECG, non-invasive blood pressure and 
oxygen saturation), preoperative vital signs were recorded in operation theatre. Intravenous line was established with 20G branula on left hand and Ringer's Lactate solution 4-6ml/kg was administered before surgery. Group K patients were premedicated with IV ketamine $0.75 \mathrm{mg} / \mathrm{kg} 30$ seconds before administration of IV induction agent while group $\mathrm{C}$ patients did not receive any premedication. After adequate preoxygenation, induction was accomplished by using injection propofol $2 \mathrm{mg} / \mathrm{kg}$ and injection atracurium $0.5 \mathrm{mg} / \mathrm{kg}$ in both the groups. Endotracheal tube of suitable size was passed and patient maintained on intermittent positive pressure ventilation with tidal volume $10 \mathrm{ml} / \mathrm{kg}$ and respiratory rate 13 breaths per minute. General anesthesia was maintained with $50 \% \mathrm{O} 2,50 \% \mathrm{~N} 2 \mathrm{O}$ and 1-2MAC sevoflurane. Heart rate and ECG was recorded $30 \mathrm{sec}$ before and continuously after traction on extraocular muscles was applied up till the end of surgery for any drop in HR or presence of arrhythmias. A decrease in HR of equal to or more than $20 \%$ on pulse oximetry and ECG or presence of arrhythmias in the form of ventricular ectopy, ventricular fibrillation or AV block on ECG was documented. Percentage change in heart rate was calculated as \%age change in $\mathrm{HR}=\mathrm{HR}$ after traction-baseline $\mathrm{HR} /$ baseline $\mathrm{HR} \times 100 \%$

In patients with $\mathrm{HR}<50$ beats/min, injection atropine $0.01 \mathrm{mg} / \mathrm{kg}$ was administered if bradycardia was not resolved within 20 s after traction on extraocular muscles is released. At the end of surgery, injection neostigmine $0.04 \mathrm{mg} / \mathrm{kg}$ and injection atropine $0.01 \mathrm{mg} / \mathrm{kg}$ were given to reverse the neuromuscular blockade. After the patients maintained adequate spontaneous breathing, they were extubated. Patients stayed in PACU for 1 hour after which they were shifted to ward.

Data analysis: collected data was entered to SPSS version 15 and was analyzed. Qualitative data such as gender or arrhythmias was presented as frequency and percentages. Age and heart rate was presented as mean $\pm \mathrm{SD}$. Comparison of two groups $\mathrm{C}$ and $\mathrm{K}$ was done by applying Chi-square test. Data was stratified for age, gender, ASA I/II, BMI. Chi-square test was used taking p-value < 0.05 as significant.

\section{RESULTS}

The study was carried out on a total of 60 patients with 30 in each group. The patients of Group K were premedicated with ketamine while patients of Group C were not given any premedication. Overall the mean age of cases in this study was $18.48 \pm 4.77$ years while mean age in Group $C$ and $K$ was $19.83 \pm 4.15$ years and $17.13 \pm 5.03$ years respectively (Table -1$)$. There were 31 male $(51.7 \%)$ and 29 female $(48.3 \%)$ cases in this study. In Control group, $18(60 \%)$ male and $12(40 \%)$ female cases were present while Ketamine group comprised of $13(43.33 \%)$ male and $17(56.67 \%)$ female cases (Fig- 1$)$.

In control group, $15(50 \%)$ cases had arrhythmia and $15(50 \%)$ cases did not develop arrhythmia while in Ketamine group, $4(13.33 \%)$ cases had arrhythmia and $26(86.67 \%)$ cases did not develop arrhythmia (Fig-2). The mean heart rate in Control group was $118.77 \pm 6.92$ / minute and in Ketamine group the mean heart rate was $101.57 \pm 15.65$ / minute (Table -2). In Control group, Oculocardiac reflex did not occur in $7(23.3 \%)$ cases only while $23(76.7 \%)$ cases had Oculocardiac reflex while in Ketamine group, there were $24(80 \%)$ cases in which Oculocardiac reflex was prevented while in $6(20 \%)$ cases Oculocardiac reflex was not prevented. The frequency of Oculocardiac reflex prevention was statistically higher in Ketamine group when compared with control group, p-value $<0.05$ (Table -3$)$.

Table 1: Descriptive statistics of age (years) in both study groups

\begin{tabular}{|l|c|c|c|c|}
\hline Study groups & Mean & S.D & Min. & Max. \\
\hline Control group & 19.83 & 4.15 & 10 & 25 \\
\hline Ketamine group & 17.13 & 5.03 & 10 & 25 \\
\hline Total & 18.48 & 4.77 & 10 & 25 \\
\hline
\end{tabular}

Table 2: Descriptive statistics of heart rate in both study groups

\begin{tabular}{|l|l|l|l|l|}
\hline Study groups & Mean & S.D & Min. & Max. \\
\hline Control group & 118.77 & 6.92 & 108.00 & 130.00 \\
\hline Ketamine group & 101.57 & 15.65 & 80.00 & 130.00 \\
\hline Total & 110.17 & 14.80 & 80.00 & 130.00 \\
\hline
\end{tabular}

Table 3:Frequency prevention of oculocardiac reflex in both groups

\begin{tabular}{|l|l|l|l|}
\hline \multirow{2}{*}{$\begin{array}{l}\text { Prevention } \\
\text { Oculocardiac reflex }\end{array}$} & \multicolumn{2}{|c|}{ Groups } & \multirow{2}{*}{ Total } \\
\cline { 2 - 4 } & Control & Ketamine & \\
\hline Yes & $7(23.3 \%)$ & $24(80 \%)$ & $31(51.7 \%)$ \\
\hline No & $23(76.7 \%)$ & $6(20 \%)$ & $29(48.3 \%)$ \\
\hline Total & $30(100 \%)$ & $30(100 \%)$ & $60(100 \%)$ \\
\hline
\end{tabular}

Fig-1: Gender distribution of cases in both groups

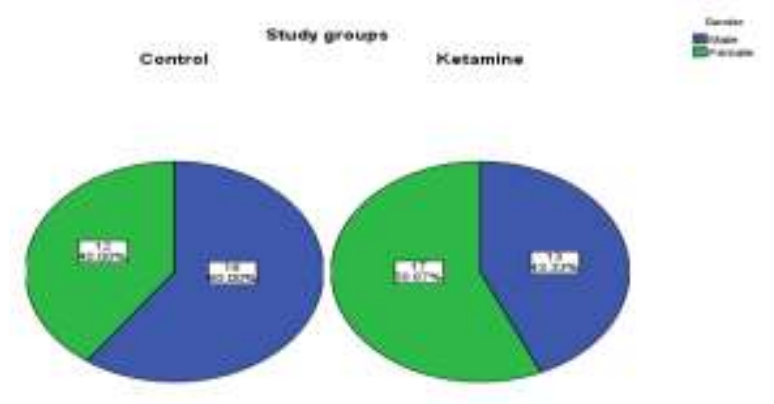

Fig-2: Frequency distribution of arrhythmias in both groups
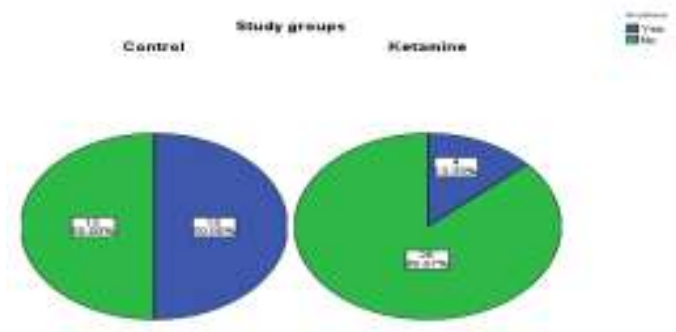

\section{DISCUSSION}

Different methods have been used to block OCR which include maintaining adequate anesthetic dephth, normoxia, normocapnia and anticholinergic premedication. However, none of them have proven completely effective ${ }^{23} .90 \%$ of patients who don't receive anticholinergic premedication develop OCR. This can be reduced to $50 \%$ by using atropine or glycopyrrolate, atropine being more effective ${ }^{18}$. OCR can also be inhibited by retrobulbar block with $2 \%$ xylocaine alongwith atropine premedication as demonstrated by Misurya et $\mathrm{al}^{19}$. Moreover, Gupta et al revealed lower occurence and severity of OCR in children receiving peribulbar block with $0.25 \%$ bupivacaine ${ }^{20}$.

Ketamine is an anaesthetic agent with intrinsic analgesic and amnestic properties. Ketamine activity is due to NMDA receptor antagonism $^{21}$. Ketamine also causes the sympatho-neuronal release of norepinephrine. Positive chronotropic effects of ketamine are due to central inhibition of efferent cardiac vagal drive and not due to baroreflex function ${ }^{22}$.

In current study, in control group Oculocardiac reflex did not occur in $7(23.3 \%)$ cases only while in Ketamine group, there were $124(80 \%)$ cases in which Oculocardiac reflex was prevented. The frequency of Oculocardiac reflex prevention was statistically higher in Ketamine group in comparison to control group, $p$-value $<0.05$. 
The usefulness of ketamine is also reported by another study i.e. occurrence of OCR in control group is $63 \%$ and in ketamine group is $20 \% .^{1}$ This survey consisted of 90 patients (aged 4-10 years) divided into three groups. Ketamine was given to Group K patients as an induction agent, Group A was given intravenous atropine as premedication and Group C was not given any premedication preoperatively. This survey revealed that induction, in the patients of squint surgery, with ketamine effectively blunts OCR and blocks any harmful effects of dysrhythmias during eye surgery when compared with atropine or control group ${ }^{1}$.

Similarly, Choi SH et al studied in 2007 that the occurrence of OCR was remarkably lower in the ketamine groups [4/40 in Group K1 (ketamine 1mg/kg) and 1/40 in Group K2 (ketamine $2 \mathrm{mg} / \mathrm{kg}$ )] while the incidence was $14 / 40$ in propofol group. Hence, intravenous ketamine 1 or $2 \mathrm{mg} / \mathrm{kg}$ on induction results in a lower incidence of OCR as compared to propofol when used with sevoflurane in children undergoing strabismus surgery ${ }^{9}$. This study is comparable with our research.

Recently, a trial was carried out by Kamal Al-Den et al that had similar results to our study. This clinical trial studied the impact of atropine and ketamine on oculocardiac reflex during strabismus surgery. They concluded that incidence of OCR is reduced significantly on induction with ketamine $1 \mathrm{mg} / \mathrm{kg}$ in comparison with atropine \& control groups which showed significant decrease in PONV ${ }^{3}$.

Similarly, Mizark et al performed a clinical trial in 2010 to correlate the consequences of intravenous ketamine infusion(1$3 \mathrm{mg} / \mathrm{kg} / \mathrm{hr}$ ) and propofol infusion $(6-9 \mathrm{mg} / \mathrm{kg} / \mathrm{hr}$ ) in children undergoing strabismus surgery. The results showed that by using anesthetics $(P=0.0001)$ and antiemetics $(P=0.004)$, occurrence of oculocardiac reflex $(P=0.02)$ was less in ketamine group. This was in agreement to our study. Moreover, the time for recovery $(P=0.008)$, agitation score in postoperative area $(P=0.005)$, Face Pain Scale $(P=0.001)$, Ramsay Sedation Score $(P=0.01)$ during awakening and at $30^{\text {th }}$ minute postoperatively $(P=0.02)$ was also lower with ketamine. So, it can be concluded that ketamine infusion is more beneficial than the infusion of propofol in children undergoing strabismus surgery ${ }^{23}$. We did not, however observe all these parameters as we purely aimed to see the incidence of OCR

In agreement to our study, Hahnenkamp et al in 2000 conducted a clinical trial to find out the result of various anesthetic regimens on OCR in pediatric patients undergoing strabismus surgery. The different anesthetic agents used were propofol+alfentanil, sevoflurane+N2O, ketamine+midazolam and halothane+N2O. All patients developed OCR except only $22 \%$ developed it in ketamine group. Median HR and BP change and incidence of atrioventricular rhythm disorders was less with ketamine. Hence, like the results of our study, it was concluded that ketamine results in minimum haemodynamic changes caused by $\mathrm{OCR}^{17}$.

Safavi et al compared the effects of thiopental, propofol and ketamine on OCR during strabismus surgery. The study concluded that mean HR was lower in patients receiving propofol as compared to patients receiving ketamine and OCR incidence was reduced in patients receiving ketamine as compared to patients receiving propofol or thiopental. Hence, just as in our study, they concluded that induction with ketamine results in minimum cardiovascular changes triggered by OCR during strabismus surgery ${ }^{14}$.

Another clinical trial produced the same results as ours which was conducted by Gourdarzi et al in 2019 to assess the outcome of pre-emptive dose of IV ketamine $(0.15 \mathrm{mg} / \mathrm{kg})$ on OCR in pediatric patients undergoing strabismus surgery. Results revealed that $21(70 \%)$ patients in control group and $10(33.3 \%)$ patients in ketamine group had OCR, the difference being significant $(P: 0.004)^{13}$. Therefore, the result of this study was consistent with ours.

In contrast to our study, Oh JN et al conducted a survey in 2012 to correlate the end-results of ketamine and midazolam on OCR in pediatric strabismus surgery. Incidence of OCR (10\% vs.
$19.4 \%$ ) between the two groups was same. The incidence of PONV (6.7 vs. $9.7 \%)$ and EA (30\% vs. $22.6 \%)$ was also similar 8 . They concluded that the incidence of OCR was not reduced by ketamine as compared with midazolam in pediatric strabismus surgery. Furthermore, the risk of PONV and EA is not increased by ketamine. Therefore, it is safe to use ketamine during pediatric strabismus surgery 4 .

Liu $Y$ et al compared the effect of sevoflurane-nitrous oxide inhalation anaesthesia with LMA and propofol-ketamine IV anaesthesia in strabismus surgery and concluded, as opposed to our study, that OCR was remarkably lower in patients receiving inhalational anesthesia as compared to TIVA group $(P<0.01)^{24}$. This could be explained by cardiodepressant effect of propofol when added to ketamine.

\section{CONCLUSION}

It is reported that Oculocardiac reflex can be prevented after premedication with low-dose intravenous ketamine during strabismus surgery. Hence, Ketamine may be used to avoid this condition. So, by minimizing the risk of OCR the risk of sinus bradycardia, ventricular ectopy, AV block, ventricular fibrillation and asystole can be avoided.

Limitations of study: The relation between type of EOM manipulated and severity of OCR was not studied. Further studies can be done to find out the effect of type of EOM manipulated and the degree of OCR. Moreover, the sample size was also limited which could disturb the exact outcome.

\section{Conflict of interest: $\mathrm{Ni}$}

\section{REFERENCES}

1. Espahodi E, Sanatkar M, Sadrossadat H, et al. Ketamine or atropine: which one better prevents oculocardiac reflex during eye surgery? A prospective randomized clinical trial. Acta Med Iran. 2015;53:158-161.

2. Subhedar R, Borse $\mathrm{Y}$, Patel S. Oculocardiac reflex during strabismus surgery in paediatric patients: A randomized case-control study. Int J Sci Stud. 2015;3(9):70-73.

3. KAMAL AL-DEN A. HEIKL, M.D., F MONA R. EL-GHAMRY, M D , Y Comparative Study between Atropine and Ketamine for Prevention of Oculocardiac Reflex in Children Undergoing Strabismus Surgery. The Medical Journal of Cairo University. 2018; 86(March): 311-317

4. Oh JN, Lee SJ, Kim SH, et al. Effect of ketamine and midazolam on oculocardiac reflex in paediatric strabismus surgery. Korean J Anesthesiol. 2013;64:500-504

5. Rodgers A, Cox RG. Anesthetic management for pediatric strabismus surgery: Continuing professional development. Can J Anaesth. 2010;57:602-617.

6. Dell R, Williams B. Anaesthesia for strabismus surgery: a regional survey. Br J Anaesth. 1999;82:761-763.

7. Barash PG. Clinical anesthesia: Lippincott Williams \& Wilkins; 2009

8. Shah TM, Samad R, Saalahuddin M, et al. Oculocardiac reflex in strabismus surgery under general anaesthesia. J Med Sci. 2013;21:84-87.

9. Choi S, Lee S, Kim S, Kim J, Kwon H, Shin Y, et al. Single bolus of intravenous ketamine for anesthetic induction decreases oculocardiac reflex in children undergoing strabismus surgery. Acta Anaesthesiol Scand. 2007;51(6):759-62

10. Ohashi T, Kase M, Yokoi M. Quantitative analysis of the oculocardiac reflex by traction on human extraocular muscle. Invest Ophthalmol Vis Sci 1986; 27: 1160-4

11. Gilani MT, Shari M, Naja MN, et al. Oculocardiac reflex during strabismus surgery. Rev Clin Med. 2016;3(1):4-7.

12. Rodgers A, Cox RG. Anesthetic management for pediatric strabismus surgery: continuing professional development. Can J Anaesth. 2010;57(6):602-17.

13. Goudarzi M, Mehrpour M, Rajabi MS, Sanatkar M, Espahbodi E, Akbari MR. The Effect of Pre-Emptive Dose of Intravenous Ketamine on Oculocardiac Reflex in Pediatric Strabismus Surgery: A Randomized Clinical Trial. Acta Med Iran. 2019;57(5):295-298.

14. Safavi M. and Honarmand A.: Comparison of effects of thiopental, propofol or ketamine on the cardiovascular responses of the oculocardiac reflex during strabismus surgery. Journal of Research in Medical Sciences, 12 (5): 246-50, 2007.

15. White PF, Way WL, Trevor AJ. Ketamine - its pharmacology and therapeutic uses. Anesthesiology. 1982;56:119-136 
16. Saarnivaara L. Comparison of thiopentone, Althesin, and ketamine in anaesthesia for otolaryngological surgery in children. $\mathrm{Br} J$ Anaesth. 1977; 49:363-370.

17. Hahnenkamp K, HÖNEMANN CW, Fischer LG, Durieux ME Muehlendyck $\mathrm{H}$, Braun $\mathrm{U}$. Effect of different anaesthetic regimes on the oculocardiac reflex during paediatric strabismus surgery. Paediatr Anaesth. 2000;10(6):601-8.

18. Mirakhur RK, Jones CJ, Dundee JW, et al. I.m. or I.V. atropine or glycopyrrolate for the prevention of oculocardiac reflex in children undergoing squint surgery. Br J Anaesth. 1982;54(10):1059-1063

19. Misurya VK, Singh SP, Kulshrestha VK. Prevention of oculocardiac reflex (O.C.R) during extraocular muscle surgery. Indian $J$ Ophthalmol. 1990;38:85-87.

20. Gupta N, Kumar R, Kumar S, Sehgal R, Sharma KR. A prospective randomised double blind study to evaluate the effect of peribulbar block or topical application of local anaesthesia combined with general anaesthesia on intra-operative and postoperative complications during paediatric strabismus surgery. Anaesthesia. 2007;62:1110-1113.

21. Trujillo KA, Smith ML, Sullivan B, Heller CY, Garcia C, Bates M. The neurobehavioral pharmacology of ketamine: implications for drug abuse, addiction, and psychiatric disorders. ILAR J. 2011;52(3):36678.

22. Morris C, Perris A, Klein J, Mahoney P. Anaesthesia in haemodynamically compromised emergency patients: does ketamine represent the best choice of induction agent? Anaesthesia. 2009;64(5):532-9.

23. Mizrak A, Erbagci I, Arici T, et al. Ketamine versus propofol for strabismus surgery in children. Clin Ophthalmol. 2010;4:673-679

24. Liu Y, Zeng QY. [Sevoflurane-N2O inhalation anaesthesia with laryngeal mask airway and propofol-ketamine intravenous anaesthesia in strabismus surgery]. Zhong Nan Da Xue Xue Bao Yi Xue Ban. 2006 Feb;31(1):97-9 